Gastroenterologe 2014 · 9:99-100

DOI 10.1007/s11377-014-0887-2

Online publiziert: 20. Februar 2014

(c) Springer-Verlag Berlin Heidelberg 2014
F. Schmitz ${ }^{1}$. G. Gerken ${ }^{2}$

${ }^{1}$ Medizinische Klinik II mit den Schwerpunkten Gastroenterologie, Onkologie/Palliativmedizin, Rheumatologie, Infektiologie und Diabetologie, Klinikum Hildesheim GmbH, Hildesheim

2 Klinik für Gastroenterologie und Hepatologie, Zentrum für Innere

Medizin, Medizinische Universitätsklinik Essen

\title{
Rationale Labordiagnostik in der Gastroenterologie
}

Die Labordiagnostik versinnbildlicht wie kaum ein anderer Bereich in der $\mathrm{Me}$ dizin - die ökonomischen Grenzen der modernen Medizin in unserem Gesundheitswesen auf einer arbeitstäglichen $\mathrm{Ba}$ sis. Vor diesem Hintergrund gehört die Kenntnis einer elaborierten Labordiag nostik zum unverzichtbaren Repertoire eines jeden Gastroenterologen im Zeitalter der molekularen Medizin; aber die rationale Diagnostik erschließt sich erst durch die kritische Würdigung der Indikationsstellungen und die Kenntnis der möglichen Aussagekraft eines Testverfahrens.

In dem vorliegenden Themenheft liefern namhafte Autoren aus dem Bereich der gastroenterologischen Universitätsund Spitzenmedizin in Deutschland für ausgewählte Szenarien einen konzisen, systematischen und ebenso pragmatischen Ansatz zum Einsatz der Labordiagnostik in der Gastroenterologie.

Eine Erhöhung der Leberwerte ist ein häufiger Grund für eine weiterreichende Labordiagnostik, um behandlungsbedürftige Lebererkrankungen frühzeitig zu erkennen und Folgeerkrankungen zu vermeiden. Blomeyer und Kollegen stellen einen diagnostischen Algorithmus zur Abklärung erhöhter Leberwerte vor, der zu einer suffizienten Diagnosestellung führt und unnötige Untersuchungen vermeiden hilft. Dabei gilt, dass eine weiterreichende Diagnostik bei erhöhten Leberwerten dann indiziert ist, wenn diese im Allgemeinen

- länger als 6 Monate bestehen,

- symptomverursachend bewertet werden oder
- die dreifache Norm überschreiten.

Die in der Basisdiagnostik erhobenen Leberwerte gestatten bereits eine Unterscheidung parenchymatöser Schäden (hepatitische Laborkonstellation) von Schäden der biliären Exkretion (cholestatische Laborkonstellation). Eine sinnvolle weitere Differenzierung erfolgt dann auf der Basis klinischer Zeichen und anamnestischer Kriterien, die als „Vortest-Wahrscheinlichkeit“ die Aussagekraft der Leberdiagnostik erheblich verbessern.

Kahraman und Herzer zeigen am Beispiel der Lebertransplantationen prinzipielle und spezifische Indikationen für eine labordiagnostische Überwachung eines Transplantierten auf. Die Indikation zur Laboranalytik zielt

- auf die Bewertung der Transplantatfunktion,

- auf die Identifikation von opportunistischen Infektionen,

- auf das therapeutische Drug-Monitoring der eingesetzten Immunsuppressiva.

Die spezifische Laboranalytik trägt der zur Transplantation führenden Lebererkrankung besondere Rechnung.

Klag und Mitarbeiter bewerten die Möglichkeiten zur Labordiagnostik in Blut und Stuhl bei den chronisch-entzündlichen Darmerkrankungen. Bei chronisch-entzündlichen Darmerkrankungen ist die Labordiagnostik für die Etablierung der Erstdiagnose, die Beurteilung der extraintestinalen Manifestationen und die Bewertung von Mangelerscheinungen sowie Therapiekomplika- tionen von besonderer Bedeutung. Dennoch wird deutlich, dass die alleinige Beurteilung chronisch-entzündlicher Darmerkrankungen durch Labormarker derzeit nicht möglich scheint. Relevante Therapieentscheidungen und Diagnosesicherung basieren auch weiterhin aus der $\mathrm{Zu}$ sammenschau klinischer, bildgebender und eben der vorgestellten labordiagnostischen Parameter.

Wiesweg und Schuler setzen sich mit den Grenzen und Möglichkeiten der Laboranalytik in der gastrointestinalen Onkologie auseinander.

\section{(>) Für die Umsetzung einer ziel-} gerichteten Therapie ist vor allem die molekulare Tumordiagnostik prognostisch von hoher Relevanz.

Die Wirksamkeit des Therapieprinzips der Neutralisation des Wachstumsfaktors EGF („Epidermal Growth Factor“) durch die monoklonalen Antikörper Cetuximab oder Panitumumab kann bei kolorektalem Karzinom auf der Basis von Mutationsanalysen im Tumorgewebe prädiktiv prognostiziert werden. Aber auch die zu erwartenden Toxizitäten handelsüblicher Zytostatika sind durch die Analyse relevanter Genvarianten labordiagnostisch vorherzusagen.

Das hepatozelluläre Karzinom (HCC) ist weltweit der fünfthäufigste Tumor. In Deutschland ist eine Zunahme der Inzidenz des HCC um $25 \%$ in den letzten 10 Jahren zu verzeichnen. Neben einer weiteren Zunahme der HCV-Infektionen wird auch eine Häufung der nichtalkoholischen Fettleberhepatitis postu- 
liert. J.F.Schlaak setzt sich kritisch mit dem Nutzen von Tumormarkerbestimmungen beim HCC auseinander. Insbesondere die Kombination der verfügbaren Marker AFP-L3 und DCP scheint die Erkennung früher Tumorstadien zu verbessern. Und weitere Testverfahren sind in der Entwicklung.

Fazit: Die Labordiagnostik in der Gastroenterologie ist ein unverzichtbares Diagnostikum in Diagnosefindung, Verlaufsbeobachtung und im Therapiemonitoring bei benignen, entzündlichen, neoplastischen und immunologischen Krankheitsbildern von Leber, Magen und Darm. Nur die präzise Kenntnis der Indikationsstellungen und der Aussagekraft der einzelnen Testverfahren ermöglicht den rationalen Einsatz dieser „Wunderwaffen“ und verhindert einen unnötigen Ressourcenverbrauch. Der aktuelle und zukünftige Weg ist in dem vorliegenden Heft direkt aufgezeigt. Der Leser darf sich über die schier unbegrenzten Möglichkeiten in diesem spannenden Entwicklungsfeld in der Gastroenterologie freuen.

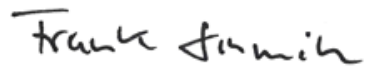

F. Schmitz

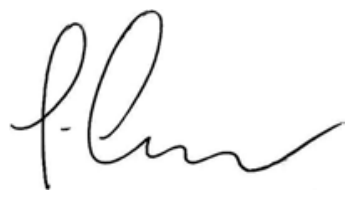

G. Gerken

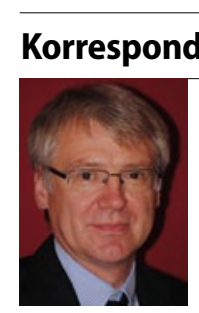

Medizinische Klinik II mit den

Schwerpunkten Gastroenterologie, Onkologie/Palliativmedizin, Rheumatologie, Infektiologie und Diabetologie, Klinikum Hildesheim GmbH

Senator-Braun-Allee 33,

31135 Hildesheim

f.schmitz@

klinikum-hildesheim.de

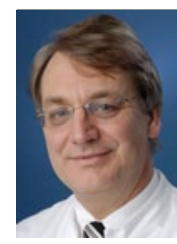

Prof. Dr. G. Gerken

Klinik für Gastroenterologie und Hepatologie, Zentrum für Innere Medizin, Medizinische Universitätsklinik Essen

Hufelandstr. 55, 45122 Essen guido.gerken@uk-essen.de

Interessenkonflikt. F. Schmitz und G. Gerken geben an, dass kein Interessenkonflikt besteht.

\section{Intoxikationen}

Als Ursache nichttraumatisch bedingter Komas stehen sowohl klinisch als auch präklinisch Intoxikationen an erster Stelle.

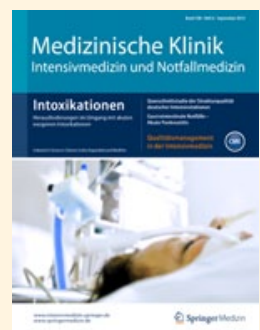
Dabei stellen besonders die ständig neuen Substanzen/Drogen und Medikamente eine große Herausforderung für den behandelnden Arzt dar.

Neben dem Wunsch für jede Intoxikation das passende Antidot zu finden, steht oft auch das Verlangen nach einer allumfassenden Analytik. Lesen Sie im Leitthemenheft "Intoxikationen" (Ausgabe 06/13) von Medizinische Klinik Intensivmedizin und Notfallmedizin über die Schwerpunkte im Umgang mit akut exogener Intoxikation und halten Sie sich auf dem aktuellen Stand über:

- Erstdiagnose und Erstbehandlungen von Vergiftungen

- Antidote in der klinischen Toxikologie

- Toxikologische Analytik

- Dermale und inhalative Intoxikationen

- Droge ist nicht gleich Droge

Bestellen Sie diese Ausgabe zum Preis von 33,-. Euro zzgl. Versandkosten bei Springer Customer Service Center Kundenservice Zeitschriften Haberstrasse 7 69126 Heidelberg Tel.: +49 6221-345-4303 Fax: +49 6221-345-4229

E-Mail: leserservice@springer.com

Suchen Sie noch mehr zum Thema? Mit e.Med, dem Online-Paket von Springer Medizin, können Sie schnell und komfortabel in über 500 medizinischen Fachzeitschriften recherchieren. Weitere Infos unter: springermedizin.de/eMed 